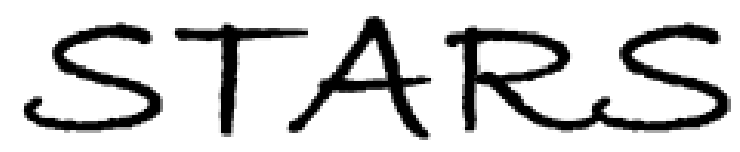

University of Central Florida

STARS

$1-1-2014$

\title{
Wide range optofluidically tunable multimode interference fiber laser
}

J E. Antonio-Lopez

University of Central Florida

J J. Sanchez-Mondragon

P. LiKamWa

University of Central Florida

D A. May-Arrioja

Find similar works at: https://stars.library.ucf.edu/facultybib2010

University of Central Florida Libraries http://library.ucf.edu

This Article is brought to you for free and open access by the Faculty Bibliography at STARS. It has been accepted for inclusion in Faculty Bibliography 2010 s by an authorized administrator of STARS. For more information, please contact STARS@ucf.edu.

\section{Recommended Citation}

Antonio-Lopez, J E.; Sanchez-Mondragon, J J.; LiKamWa, P.; and May-Arrioja, D A., "Wide range optofluidically tunable multimode interference fiber laser" (2014). Faculty Bibliography 2010s. 4995. https://stars.library.ucf.edu/facultybib2010/4995

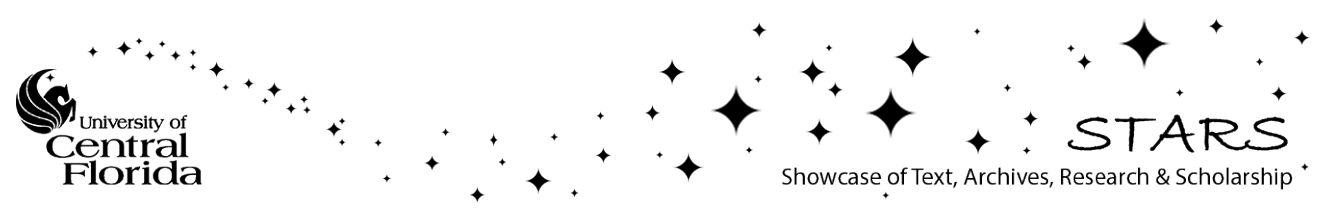


PAPER

\section{Wide range optofluidically tunable multimode interference fiber laser}

To cite this article: J E Antonio-Lopez et al 2014 Laser Phys. 24085108

View the article online for updates and enhancements.
Related content

- $\frac{\text { A stable multi-wavelength PM-EDF laser }}{\text { based on a nonlinear amplifying loop }}$
$\frac{\text { mirror and a TCF comb filter }}{\text { Hui Zou, Shuqin Lou, Wei Su et al. }}$
- Low-threshold dual-wavelength erbium-
$\frac{\text { doped fiber laser based on ring core fiber }}{\text { with core-offset structure }}$
Y H Qi, J Sun, W X Jin et al.
- Widely tunable, narrow line width and low
optical noise continuous-wave all fiber
Er:Yb co-doped double-clad ring laser
Khmaies Guesmi, Faouzi Bahloul,
Georges Semaan et al.

Recent citations

- $\frac{\text { Tunable dual-wavelength } \mathrm{Er}^{3+}{ }^{+} \text {-doped fibre }}{\frac{\text { laser based on a single multimode }}{\text { interference filter }}}$
$\mathrm{N} \mathrm{Li} \mathrm{et} \mathrm{al}$
- All-fiber widely tunable mode-locked
$\frac{\text { thulium-doped laser using a curvature }}{\text { multimode interference filter }}$
$\mathrm{N} \mathrm{Li} \mathrm{et} \mathrm{al}$
- Compact wavelength-tunable actively Q-
$\underline{\text { switched fiber laser in CW and pulsed }}$
operation based on a fiber Bragg arating
A González-García et al




\title{
Wide range optofluidically tunable multimode interference fiber laser
}

\author{
J E Antonio-Lopez ${ }^{1}$, J J Sanchez-Mondragon ${ }^{2}$, P LiKamWa ${ }^{1}$ and \\ D A May-Arrioja ${ }^{3}$ \\ ${ }^{1}$ CREOL, The College of Optics and Photonics, University of Central Florida, Orlando, FL \\ 32816-2700, USA \\ 2 Instituto Nacional de Astrofísica, Óptica y Electrónica, AP 51 y 216, Tonantzintla, Puebla 72000, \\ Mexico \\ ${ }^{3}$ Fiber and Integrated Optics Laboratory, Electronics Engineering Department, UAMRR, Universidad \\ Autónoma de Tamaulipas, Reynosa, Tamaulipas 88779, Mexico \\ E-mail: darrioja@uat.edu.mx
}

Received 29 April 2014, revised 16 May 2014

Accepted for publication 16 May 2014

Published 15 July 2014

\begin{abstract}
An optofluidically tunable fiber laser based on multimode interference (MMI) effects with a wide tuning range is proposed and demonstrated. The tunable mechanism is based on an MMI fiber filter fabricated using a special fiber known as no-core fiber, which is a multimode fiber (MMF) without cladding. Therefore, when the MMI filter is covered by liquid the optical properties of the no-core fiber are modified, which allow us to tune the peak wavelength response of the MMI filter. Rather than applying the liquid on the entire no-core fiber, we change the liquid level along the no-core fiber, which provides a highly linear tuning response. In addition, by selecting the adequate refractive index of the liquid we can also choose the tuning range. We demonstrate the versatility of the optofluidically tunable MMI filter by wavelength tuning two different gain media, erbium doped fiber and a semiconductor optical amplifier, achieving tuning ranges of 55 and $90 \mathrm{~nm}$ respectively. In both cases, we achieve side-mode suppression ratios (SMSR) better than $50 \mathrm{dBm}$ with output power variations of less than $0.76 \mathrm{dBm}$ over the whole tuning range.
\end{abstract}

Keywords: erbium-doped fiber laser, tunable laser, multimode interference, multimode fiber

(Some figures may appear in colour only in the online journal)

\section{Introduction}

Tunable fiber lasers are crucial components for a variety of photonic applications ranging from fiber sensors to telecommunication systems. In the case of sensing applications, tunable lasers are employed as elements for optical testing, analysis and metrology. Unlike single wavelength lasers, the versatility offered by tunable laser configurations relies on the ability to dynamically change both their spectral and intensity features [1]. Such characteristics make tunable lasers an ideal tool for the monitoring of multiplexed sensor systems. On the other hand, optical communication systems exhibit a never-ending demand for larger bandwidths, which increases considerably every year. Dense wavelength division multiplexing (DWDM) has been successfully used to increase the telecommunication bandwidth of single mode fibers (SMF) through using different carriers, i.e. different wavelengths, along a single optical fiber. In this respect, tunable lasers play a key role in the deployment of DWDM systems because they can easily replace the need for a set of lasers with specific wavelengths, which is a cost-effective approach for such systems [2, 3]. An important feature in the vast majority of tunable lasers is that their tuning range, and ultimately the cost of the laser, depends entirely on the technology employed to manufacture the tunable filters. Therefore, in order to reduce the cost of tunable lasers we need to develop a tunable filter that is inexpensive, while achieving a wide tunable range.

Based on their operational principle, and considering only widely tunable lasers with tuning ranges beyond $50 \mathrm{~nm}$, there 
are nowadays a number of tunable lasers based on different technologies. Typical examples for wide wavelength tuning include Fabry-Perot cavities, bulk gratings, Mach-Zehnder interferometer filters, fiber Bragg gratings (FBG) and allfiber Lyot filters, just to mention a few [4-15]. Several works have been previously reported based on Fabry-Perot cavities that exhibit very broad wavelength tuning ranges up to $100 \mathrm{~nm}[4,5]$. However, to achieve such a wide tuning range it is absolutely necessary to use two or more tunable FabryPerot filters, which is a great disadvantage because the tunable laser becomes a complex and expensive system. In the case of bulk grating filters [8], even when they exhibit a very fine bandpass wavelength response, their use eliminates the all-fiber laser arrangement and makes the system more sensitive to mechanical disturbances. A two-taper Mach-Zehnder interferometer filter has also been used to achieve wide wavelength tuning in the order of $55 \mathrm{~nm}$ [9]. However, very precise mechanical movements are required to bend one of the fiber tapers, which could be a drawback for the laser when operated continuously over long time periods. FBG [10-14] and all-fiber Lyot filters [15] have also been used but their tuning range is limited to below $50 \mathrm{~nm}$. A device that has recently attracted a lot of attention for tunable laser applications is the one based on multimode interference (MMI) effects [16, 17]. The key features of MMI devices are related to their simple fabrication process, just splicing a multimode fiber (MMF) between two SMF, and the fact that they exhibit a bandpass filter response that can be used for tuning applications. Different MMI configurations have been used to tune MMI devices [18-20] but, so far, a maximum tuning range of $60 \mathrm{~nm}$ has been achieved [20]. Tuning was achieved by increasing the separation between an SMF and the MMF inside a liquid filled capillary tube, which effectively increases the length of the MMF. The main issue was related to the losses when the separation was too large, which ultimately limits the tuning range. In this work, we demonstrate a widely tunable all-fiber MMI fiber laser using an optofluidically tunable MMI filter. Wavelength tuning is achieved using an optofluidic mechanism in which a liquid moves vertically along the MMF and changes the optical properties of the fiber covered by the liquid. This is realized by using an MMF without cladding, also known as no-core fiber. Since the peak wavelength of MMI devices is directly related to the optical properties of MMF, wavelength tuning is achieved by moving the liquid along the no-core MMF. By using this MMI filter, a tunable ring cavity EDF laser was demonstrated covering a wavelength range of $55 \mathrm{~nm}$, which was mainly limited by the available gain media. However, when the gain media are replaced by a broadband semiconductor optical amplifier (SOA), a tunability of almost $90 \mathrm{~nm}$ is achieved with excellent power uniformity. We should highlight that the device is quite simple and relatively inexpensive when compared with other tuning mechanisms.

\section{Optofluidically tunable MMI fiber filter}

The concept behind the operation of an MMI filter is quite simple and has been described elsewhere [17-20]. The key

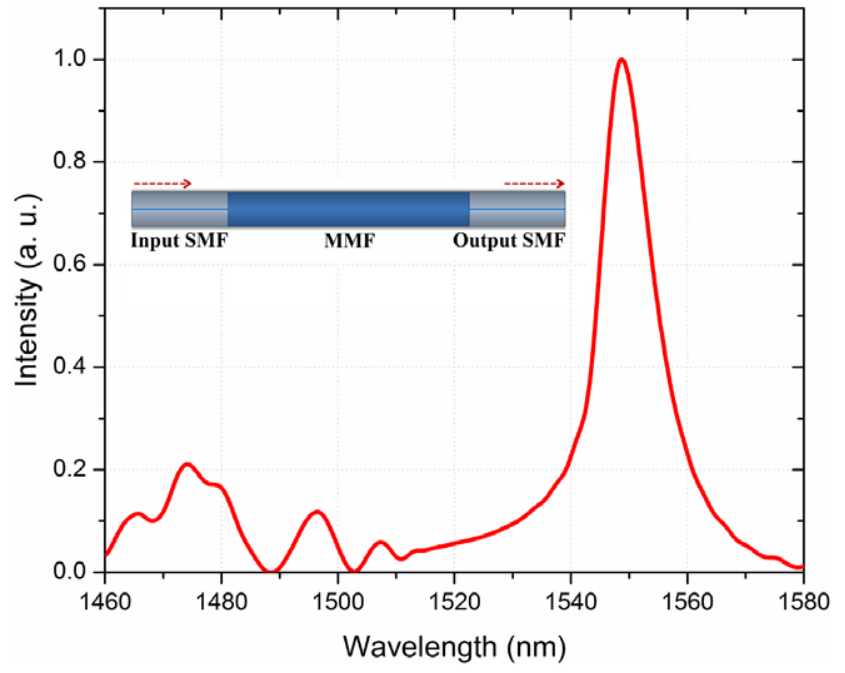

Figure 1. Spectral response of an MMI fiber filter (inset: schematic of an MMI fiber filter using no-core fiber).

element is a segment of MMF, with a precise length, which is spliced between two SMF as shown in the inset of figure 1 . The light launched into the MMF will excite the modes supported by the MMF, limited by the launching conditions, and as the modes propagate, they will form images of the input field along the MMF axis. Such images are formed when the accumulated phase difference between the modes is an integer multiple of $2 \pi$, such that the modes recombine inphase. The MMI peak wavelength is governed by the following equation,

$$
\lambda_{0}=\frac{4 n_{\mathrm{MMF}} D_{\mathrm{MMF}}^{2}}{L_{\mathrm{MMF}}},
$$

where $\lambda_{0}$ is the free space wavelength, $n_{\mathrm{MMF}}$ and $D_{\mathrm{MMF}}$ correspond to the refractive index (RI) and diameter of the fundamental mode respectively, and $L_{\mathrm{MMF}}$ is the length of the MMF. As shown in equation (1), a specific MMF length corresponds to a particular peak wavelength that exhibits maximum transmission through the MMI filter. However, if we are detuned from this wavelength the image will be formed before or after the output MMF-SMF interface and coupling to the output SMF will be reduced. Therefore, when a continuum spectrum is sent through such an MMI device, a bandpass filter response is obtained as shown in figure 1.

Based on equation (1), a tunable MMI filter can be achieved by changing the parameters of the MMF, such as the RI of the MMF (thermo-optic effect) or the effective length of the MMF as described in [14]. A closer inspection of equation (1) reveals that a larger tuning effect could be obtained, because of its square dependence, if we were able to modify the diameter of the MMF in real time. Although this is not quite obvious to achieve, we can do this by employing a special MMF fiber known as a no-core fiber, which is basically an MMF without cladding. Therefore, when the fiber is immersed in a liquid with an RI value lower than that of the no-core fiber, the properties of the MMF will be modified. In fact, both $n_{\mathrm{MMF}}$ and $D_{\mathrm{MMF}}$ are modified, but if the RI of the liquid is close to that of the no-core fiber, the dominating 


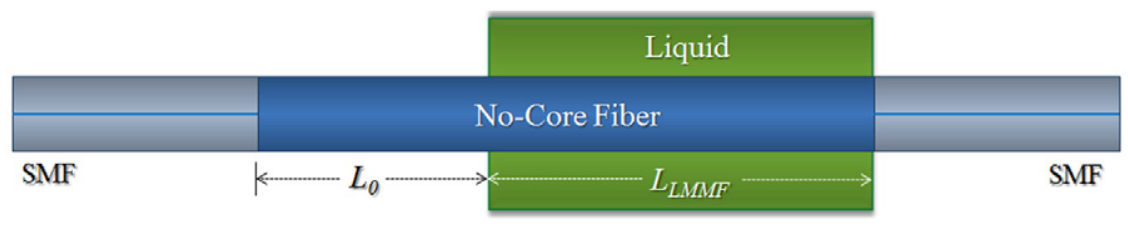

Figure 2. Schematic of the optofluidically tunable MMI fiber filter.

term will be the effective diameter. As shown in figure 2, rather than changing the RI around the entire no-core fiber length, we gradually increase or decrease the length of nocore fiber covered by the liquid. This is equivalent to having an MMI structure that is formed by two slightly different MMF. In this situation, the peak wavelength is calculated by adding the fractional phase contribution of each MMF segment. Therefore, the peak wavelength in such an MMI structure can be calculated by [21],

$$
\lambda_{0}=(4)\left[\frac{n_{\mathrm{LMMF}} D_{L \mathrm{MMF}}^{2}}{L_{T}}\left(\frac{L_{\mathrm{LMMF}}}{L_{T}}\right)+\frac{n_{\mathrm{MMF}} D_{\mathrm{MMF}}^{2}}{L_{T}}\left(\frac{L_{0}}{L_{T}}\right)\right] \text {,(2) }
$$

where $n_{\mathrm{LMMF}}$ and $D_{\mathrm{LMMF}}$ correspond to the RI and diameter of the fundamental mode and $L_{\mathrm{LMMF}}$ is the length of the no-core fiber covered with liquid. Here, $L_{0}$ is the no-core fiber section without liquid and $L_{T}$ is the total no-core fiber length. The variables in the second term of equation (2) are the same as described in equation (1) for the no-core fiber in air. As shown in equation (2), the advantage of our tunable MMI filter is that changes in the length of the no-core fiber, with and without liquid, provide a linear wavelength response while the slope is controlled by the effective RI and diameter, which is dictated by the RI of the liquid. Therefore, the largest tuning range should be obtained as the RI of the liquid approaches that of the no-core fiber because this provides maximum change of the effective RI and diameter.

The no-core fiber used in our experiments has an RI of $n=1.444$ and diameter of $125 \mu \mathrm{m}$. In order to evaluate the tuning range of our design we fabricated MMI filters with no-core fiber lengths of 58.81, 59.91 and $61.28 \mathrm{~mm}$, which correspond to peak wavelengths of 1534, 1506 and $1472 \mathrm{~nm}$ respectively. The liquids used for each MMI filter correspond to index matching liquids from Cargille ${ }^{\circledR}$ with RI values of $1.4050,1.4340$ and 1.4380 at $1550 \mathrm{~nm}$, which are lower than the RI value of 1.444 of the no-core fiber. The MMI filter was interrogated using an Agilent tunable laser with a wavelength range from 1460 to $1580 \mathrm{~nm}$, and the transmitted intensity was measured using a photodetector. The liquid level was raised in steps of $10 \mathrm{~mm}$, and the spectrum was acquired at each step. As shown in figure 3(a), when the RI of the liquid is significantly lower $(n=1.4050)$ we observe that the spectrum is red-shifted as the liquid level increased, and a total tuning range of $37.4 \mathrm{~nm}$ is obtained. We can also notice that the signal intensity also increases, which we believe is related to the fact that scattering losses are reduced when the refractive index contrast is reduced. In the case of a liquid with an RI value that is very close to that of the no-core fiber $(n=1.4380)$ we obtain the largest tuning range of $98.4 \mathrm{~nm}$ as depicted in figure $3(b)$. Since the image is formed by the in-phase combination of the propagating modes, the reduction in the signal intensity is correlated with the loss of higher order modes due to reduced index contrast between core and liquid cladding. It is worth noting that a wider tuning could be achieved by slightly increasing the RI value, but even higher losses would need to be tolerated. Based on such results, it is feasible to use a liquid with an RI value that could compensate both previous effects and obtain a wide tuning range with minimum signal intensity variation. As shown in figure $3(c)$, a liquid with an RI of $n=1.434$ provides such an effect allowing a tuning range of $68.6 \mathrm{~nm}$ with negligible signal variation. Also shown in figure $3(d)$ is the absolute wavelength shift for each liquid as a function of the liquid level around the no-core fiber. As shown in this figure, the tuning response is highly linear for each liquid. It is clear from figure $3(d)$ that a specific tuning range can be easily selected by choosing the adequate liquid RI value, which could be beneficial for some applications.

\section{Tunable fiber lasers}

In order to demonstrate the versatility of our optofluidically tunable MMI filter, two different gain media with different bandwidths were used. A tunable fiber ring laser was fabricated using $10 \mathrm{~m}$ of L-band erbium doped fiber (EDF) as the gain medium, whose schematic diagram is depicted in figure 4 (left). One end of the optofluidically tunable MMI filter was spliced to the L-band fiber while the other end was spliced to the input of a 10/90 fiber coupler. The $10 \%$ output allowed us to continuously monitor the lasing spectral response, and it was acquired by using an optical spectrum analyzer (OSA) from Agilent working at $0.06 \mathrm{~nm}$ resolution. The $90 \%$ output arm is spliced to the input of an optical isolator to keep the laser unidirectional, while the isolator output is spliced to a 980/1550 wavelength division multiplexer (WDM). The ring is closed through splicing the WDM output to the L-band EDF. A $980 \mathrm{~nm}$ wavelength laser diode with a pump power of $150 \mathrm{~mW}$ from Lucent Technologies is used to pump the L-band EDF through the WDM fiber coupler.

The MMI device was fabricated by splicing a $57.70 \mathrm{~mm}$ long section of no-core fiber between two SMF. Such a length provides a peak wavelength close to $1560.0 \mathrm{~nm}$, which sets the starting point of the tuning range. Since a tuning range close to $60 \mathrm{~nm}$ is expected from this gain medium, we choose a liquid with an RI value of 1.4300. The liquid level was increased in steps of $5 \mathrm{~mm}$, and the lasing spectrum was acquired at each step. Figure 5(a) plots the superimposed laser output spectra at different liquid levels, in which the spectra are shown for 

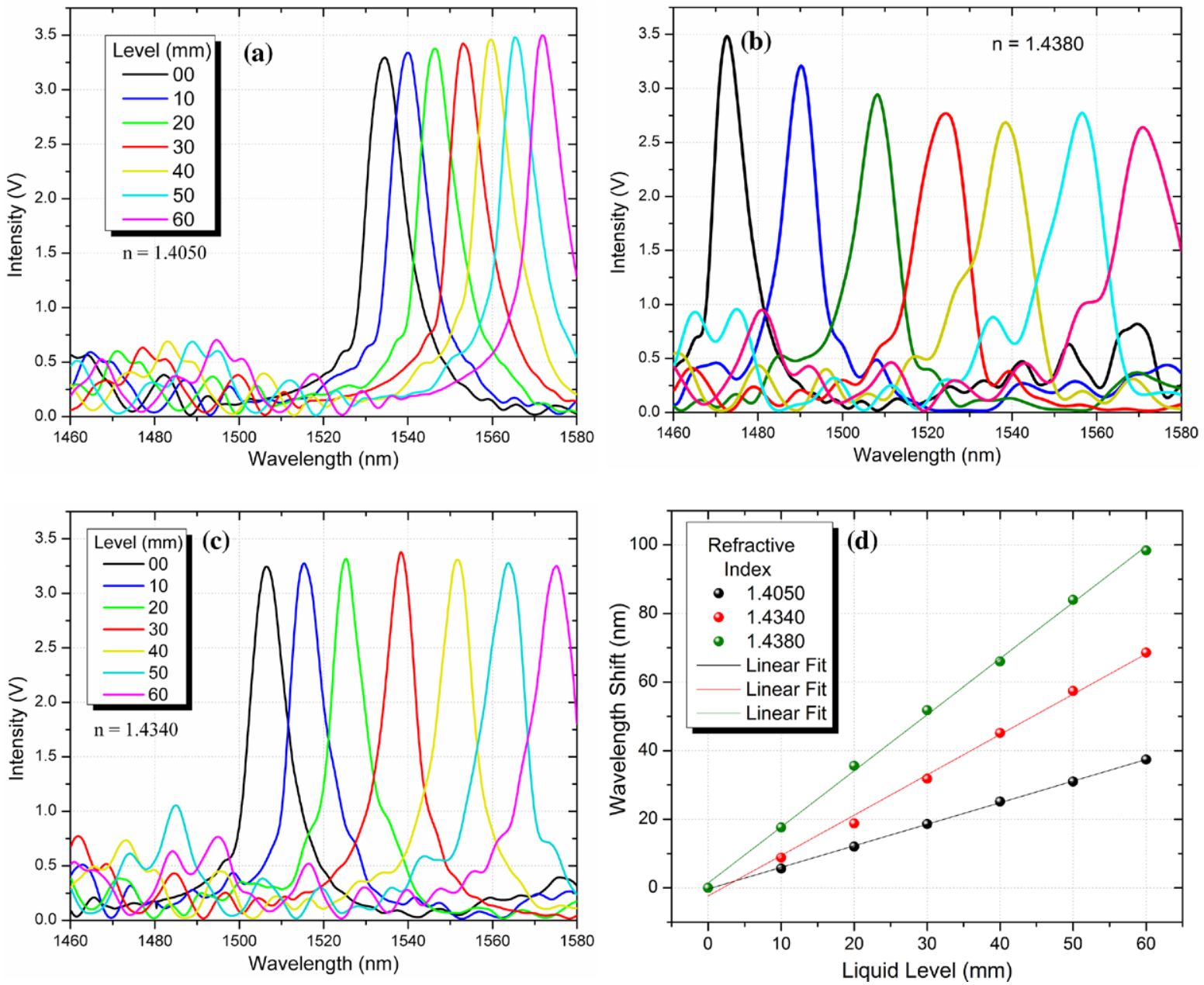

Figure 3. Spectral response of the optofluidically tunable MMI filter for different liquid levels for $(a) n=1.4050,(b) n=1.4380$, (c) $n=1.4340$, and $(d)$ total wavelength shift as a function of the liquid level for the three liquids.
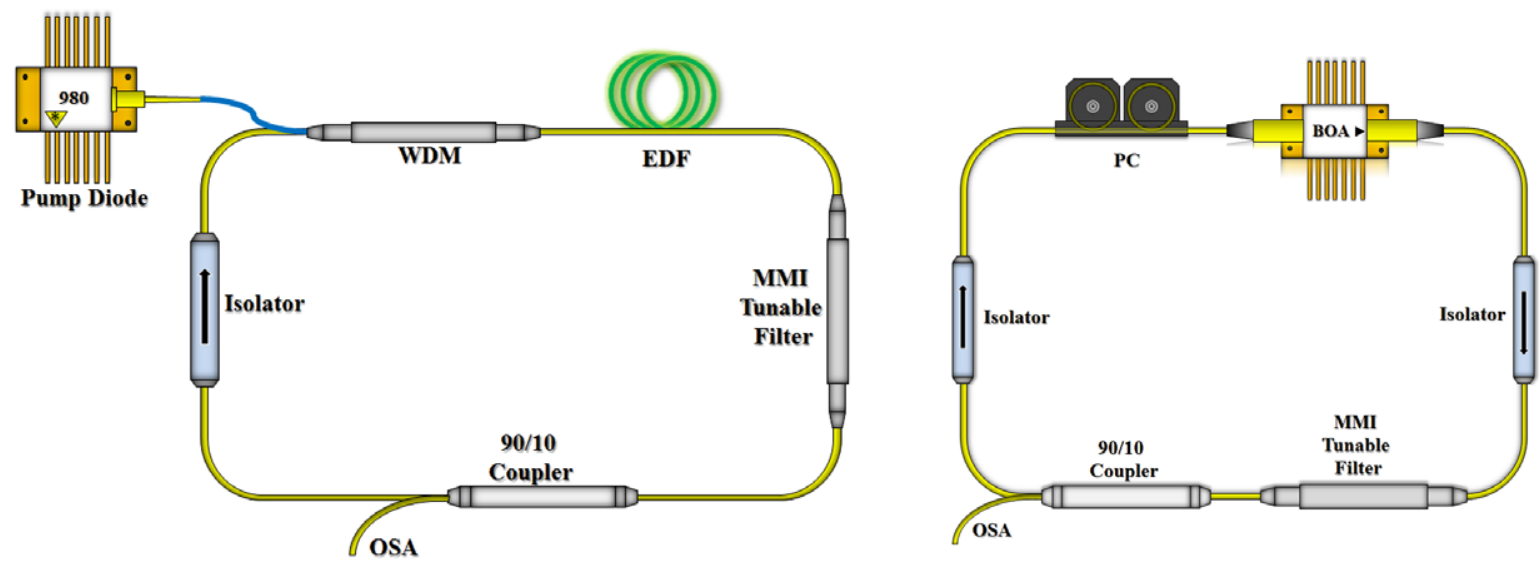

Figure 4. Experimental set-up of the optofluidically tunable MMI EDF laser (left), and experimental set-up of the optofluidically tunable MMI BOA fiber laser (right).

liquid increments of $10 \mathrm{~mm}$ for better appreciation. As shown here, the lasing line are tuned over $55 \mathrm{~nm}$ from 1559.4 to $1614.4 \mathrm{~nm}$ while the side-mode suppression ratio (SMSR) is maintained at a value exceeding $50 \mathrm{dBm}$ over the whole tuning range. The measured line width of the tunable laser output is less than $0.18 \mathrm{~nm}$. The peak wavelength as a function of the liquid level is also shown in figure $5(b)$, where we can observe a linear tuning response. Although the gain spectrum of the EDF is uneven, the output power difference among all lasing lines is less than $0.76 \mathrm{dBm}$.A broader gain medium can be achieved by replacing the gain medium with an SOA. We used the booster optical amplifier (BOA) BOA 1004 from 

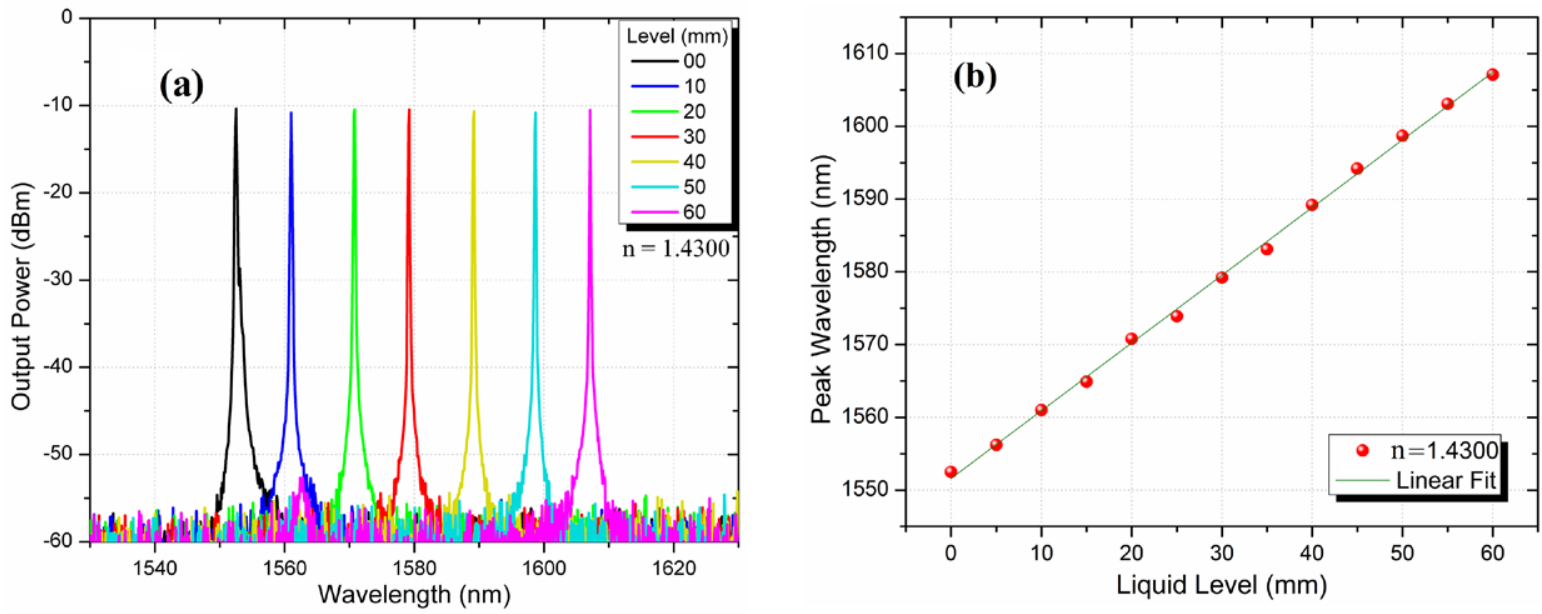

Figure 5. (a) Superimposed spectral response of the optofluidically tunable MMI EDF laser, and $(b)$ peak lasing wavelength against liquid level.
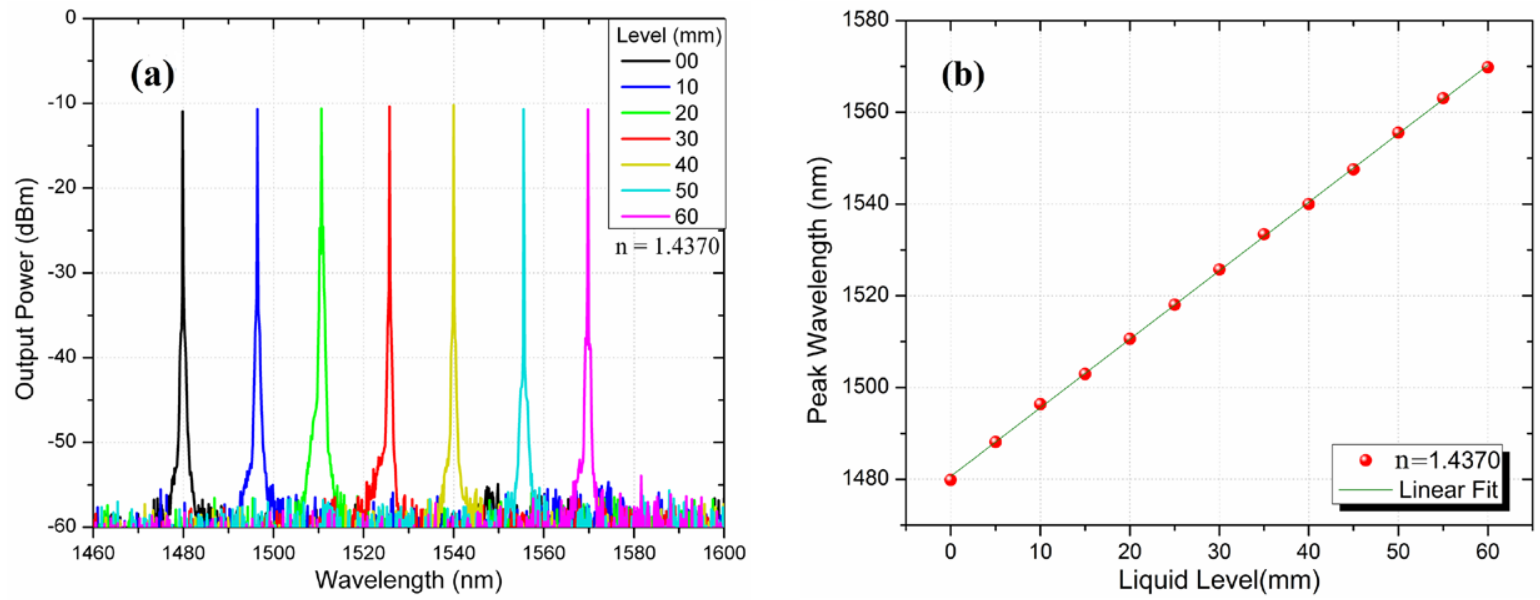

Figure 6. (a) Superimposed spectral response of the optofluidically tunable MMI BOA fiber laser, and (b) peak lasing wavelength against liquid level.

COVEGA that provided a bandwidth slightly wider than $90 \mathrm{~nm}$ and an even gain profile. As shown in figure 4 (right), the BOA replaces the pump diode, WDM and EDF, and since it has fiber pigtails, it can be easily spliced into the ring cavity. A polarization controller has to be added to the ring because the BOA is polarization dependent. Another isolator has to be included in the cavity to eliminate some resonances that are observed due to some reflections into the BOA. In this case the MMI filter consisted of a no-core length of $60.98 \mathrm{~mm}$, which corresponds to a peak wavelength of $1480 \mathrm{~nm}$, and we selected a liquid with an RI value of $n=1.437$ to obtain close to $90 \mathrm{~nm}$ tuning. As shown in figure $6(a)$, we can tune the laser over $90 \mathrm{~nm}$ while maintaining a low output power variation below $0.73 \mathrm{dBm}$. The measured line width of the tunable laser output is less than $0.07 \mathrm{~nm}$. Also shown in figure $6(b)$ is the peak wavelength against the liquid level, and again the tuning response remains highly linear. We should emphasize that even when our MMI filter is operating within the $1550 \mathrm{~nm}$ window, it could be operated at any wavelength as long as the fiber transmits light at such a particular wavelength window.
Any other gain medium that is not fiberized could make use of an external coupling scheme and take advantage of our filter capabilities.

\section{Conclusions}

We demonstrated a wide range optofluidically tunable MMI fiber laser. The advantage of this tuning mechanism is related to its wide tuning range, highly linear response, as well as the ability to select the tuning range by changing the RI of the liquid. We demonstrated the versatility of our filter by tuning two different gain media, EDF and SOA, achieving tuning ranges of 55 and $90 \mathrm{~nm}$ respectively. In both cases, we achieve an SMSR better than $50 \mathrm{dBm}$ with output power variations of less than $0.76 \mathrm{dBm}$.

\section{Acknowledgments}

We sincerely appreciate the support from the Consejo Nacional de Ciencia y Tecnología (CONACyT) under contracts 
CB-2010/157866 and CB-2008/101378. JEAL acknowledges CONACyT for their support through a postdoctoral scholarship.

\section{References}

[1] Saito M and Kikuchi K 1997 Opt. Rev. 4527

[2] Keiser G E 1999 Opt. Fiber Technol. 53

[3] Delorme F 1998 J. Quant. Electron. 341706

[4] Dong X, Ngo N, Shum P, Hwa-Yaw T and Dong X 2003 Opt. Express 111689

[5] Fu Z, Yang D, Ye W, Kong J and Shen Y 2009 Opt. Laser Technol. 41392

[6] Yamashita S and Nishihara M 2001 J. Sel. Top. Quant. Electron. 741

[7] Wei Y, Hu K, Sun B and Wang T 2012 Laser Phys. 22770

[8] Chen H, Leblanc M and Schinn G W 2003 Opt. Commun. 216119

[9] Wang X, Li Y and Bao X 2010 Opt. Lett. 353354

[10] Kamynin V A, Kablukov S I, Raspopin K S, Antipov S O, Kurkov A S, Medvedkov O I and Marakulin A V 2012 Laser Phys. Lett. 9893
[11] Lobach I A, Kablukov S I, Podivilov E V and Babin S A 2014 Laser Phys. Lett. 11045103

[12] Abdullina S R, Babin S A, Vlasov A A, Kablukov S I, Kurkov A S and Shelemba I S 2007 J. Quant. Electron. 371146

[13] Choi B K, Park I G, Byun J H, Kim N, Han S P, Park K H, Seo J K, Lee H K and Jeon M Y 2013 Laser Phys. Lett. 10125105

[14] Durán-Sánchez M, Kuzin E A, Pottiez O, Ibarra-Escamilla B, González-García A, Maya-Ordoñez F, Álvarez-Tamayo R I and Flores-Rosas A 2014 Laser Phys. Lett. 11015102

[15] Wei L, Liu L, Feng S and Mao Q 2013 Laser Phys. 23055102

[16] Soldano L B and Pennings E C M 1995 J. Light. Technol. 13615

[17] Mohammed W S, Mehta A and Johnson E G 2004 J. Light. Technol. 22469

[18] Selvas R, Torres-Gomez I, Martinez-Rios A, Alvarez-Chavez J, May-Arrioja D A, LiKamWa P, Mehta A and Johnson E 2005 Opt. Express 139439

[19] Mohammed W S, Smith P W E and Gu X 2006 Opt. Lett. 312547

[20] Castillo-Guzman A, Antonio-Lopez J E, Selvas-Aguilar R, May-Arrioja D A, Estudillo-Ayala J and LiKamWa P 2010 Opt. Express 18591

[21] Antonio-Lopez J E, Sanchez-Mondragon J J, LiKamWa P and May-Arrioja D A 2011 Opt. Lett. 363425 